\title{
A Meta-Analysis of the Association between the hOGG1 Ser326Cys Polymorphism and the Risk of Esophageal Squamous Cell Carcinoma
}

\author{
Junjie Zhang ${ }^{1,2,49}$, Jingshi Zhou ${ }^{39}$, Ping Zhang ${ }^{2}$, Weiping Wang ${ }^{2}$, Shiheng Tao ${ }^{1,4 *}$, Minghua Wang ${ }^{2 *}$ \\ 1 College of Life Science, Northwest A \& F University, Yangling, Shaanxi, China, 2 Department of Biochemical and Molecular Biology, Medical College, Soochow University, \\ Suzhou, Jiangsu, China, 3 Department of Hepatopancreaticobiliary Surgery, Xijing Hospital, the Fourth Military Medical University, Xi'an, Shaanxi, China, 4 Bioinformatics \\ Center, Northwest A \& F University, Yangling, Shaanxi, China
}

\begin{abstract}
Background: Genetic polymorphism of human 8-oxoguanine glycosylase 1 (hOGG1) Ser326Cys (rs1052133) has been implicated in the risk of Esophageal Squamous Cell Carcinoma (ESCC). However, the published findings are inconsistent. We therefore performed a meta-analysis to derive a more precise estimation of the association between the hOGG1 Ser326Cys polymorphism and ESCC risk.

Methodology/Principal Findings: A comprehensive search was conducted to identify eligible studies of hOGG1 Ser326Cys polymorphism and the risk of the ESCC. Three English and two Chinese databases were used, and ten published casecontrol studies, including 1987 cases and 2926 controls were identified. Odds ratios (ORs) and 95\% confidence intervals (Cls) were used to assess the strength of the association in the dominant and recessive model. Pearson correlation coefficient (PCC) and standard error (SE) were used to assess the number of Cys allele and ESCC risk in the additive model. Overall, significant associations between the hOGG1 Ser326Cys polymorphism and ESCC risk were found in the recessive model: $\mathrm{OR}=1.37$ (95\% Cl: 1.06-1.76, $\mathrm{p}=0.02$ ). We also observed significant associations in the Caucasian, Chinese language, population based control and tissue subgroups. In the additive model, positive correlation was found between the number of Cys allele and the risk of ESCC in overall studies $(P C C=0.109, S E=0.046, p=0.02)$, Caucasian subgroup and population subgroup. Funnel plot and Egger's test indicate there was no publication bias in this meta-analysis.

Conclusion: Under the published data, the hOGG1 Ser326Cys polymorphism is associated with ESCC risk in the recessive and additive model. Compared with the Ser/Ser and Ser/Cys genotype, Cys/Cys genotype might contribute to increased risk of ESCC. And the risk of ESCC is positively correlated with the number of Cys allele. A better case-control matched study should be designed in order to provide a more precise estimation.
\end{abstract}

Citation: Zhang J, Zhou J, Zhang P, Wang W, Tao S, et al. (2013) A Meta-Analysis of the Association between the hOGG1 Ser326Cys Polymorphism and the Risk of Esophageal Squamous Cell Carcinoma. PLoS ONE 8(6): e65742. doi:10.1371/journal.pone.0065742

Editor: Zhi Wei, New Jersey Institute of Technology, United States of America

Received December 17, 2012; Accepted April 27, 2013; Published June 6, 2013

Copyright: (c) 2013 Zhang et al. This is an open-access article distributed under the terms of the Creative Commons Attribution License, which permits unrestricted use, distribution, and reproduction in any medium, provided the original author and source are credited.

Funding: This work was supported by the grant from the National Natural Science Foundation of China (Grant 81071957) (http://www.nsfc.gov.cn). The funders had no role in study design, data collection and analysis, decision to publish, or preparation of the manuscript.

Competing Interests: The authors have declared that no competing interests exist.

*E-mail: shihengt@nwsuaf.edu.cn (ST); mhwang@suda.edu.cn (MW)

9 These authors contributed equally to this work.

\section{Introduction}

Esophageal cancer (EC) is the sixth most common cancer worldwide with 5 -year survival rate less than $10 \%$ and occurs at a relatively high frequency in certain areas of China [1,2]. Esophageal Squamous Cell Carcinoma (ESCC) is the major type of EC in China, and the mechanism of ESCG still remains unclear. Many factors may increase the risk of ESCC, including environmental and genetic factors. Several studies have found out that DNA repair efficiency in cancer patients is lower than that of normal people, and the variants of the genes involved in DNA repair can lead to increasing risk of cancer [3]. The human 8oxoguanine glycosylase 1 (hOGGl) gene, located on chromosome 3, encodes 8-hydroxygumine DNA glycosylase 1 (OGG1) that can repair damaged DNA by excising 8-dihydro-8-oxoguanine (8-OHG) [4]. Genetic variations in hOGGl gene may alter glycosylase activity, increasing the cancer risk [5]. There are several polymorphisms in the hOGG1 gene [6]; and Ser326Cys polymorphism has attracted widespread attention. With a Ser to Cys amino acid substitution at codon 326, Ser326Cys can affect the function of hOGG1. This variation maybe associated with risk of cancer. A study indicated that compared to the 326Cys variant enzyme, the 326Ser enzyme of hOGGl has a higher activity [7]. Many studies have focused on the association of Ser326Cys polymorphism in hOGG1 and ESCG risk, including several casecontrol studies [8-12]. However, the results of these studies remained inconclusive and inconsistent. In this meta-analysis, by searching individual dataset from all eligible case-control studies published to date, we aimed to estimate the role of hOGGl Ser326Cys polymorphism in the risk of ESCG as well as to quantify the between-study heterogeneity and potential bias. 


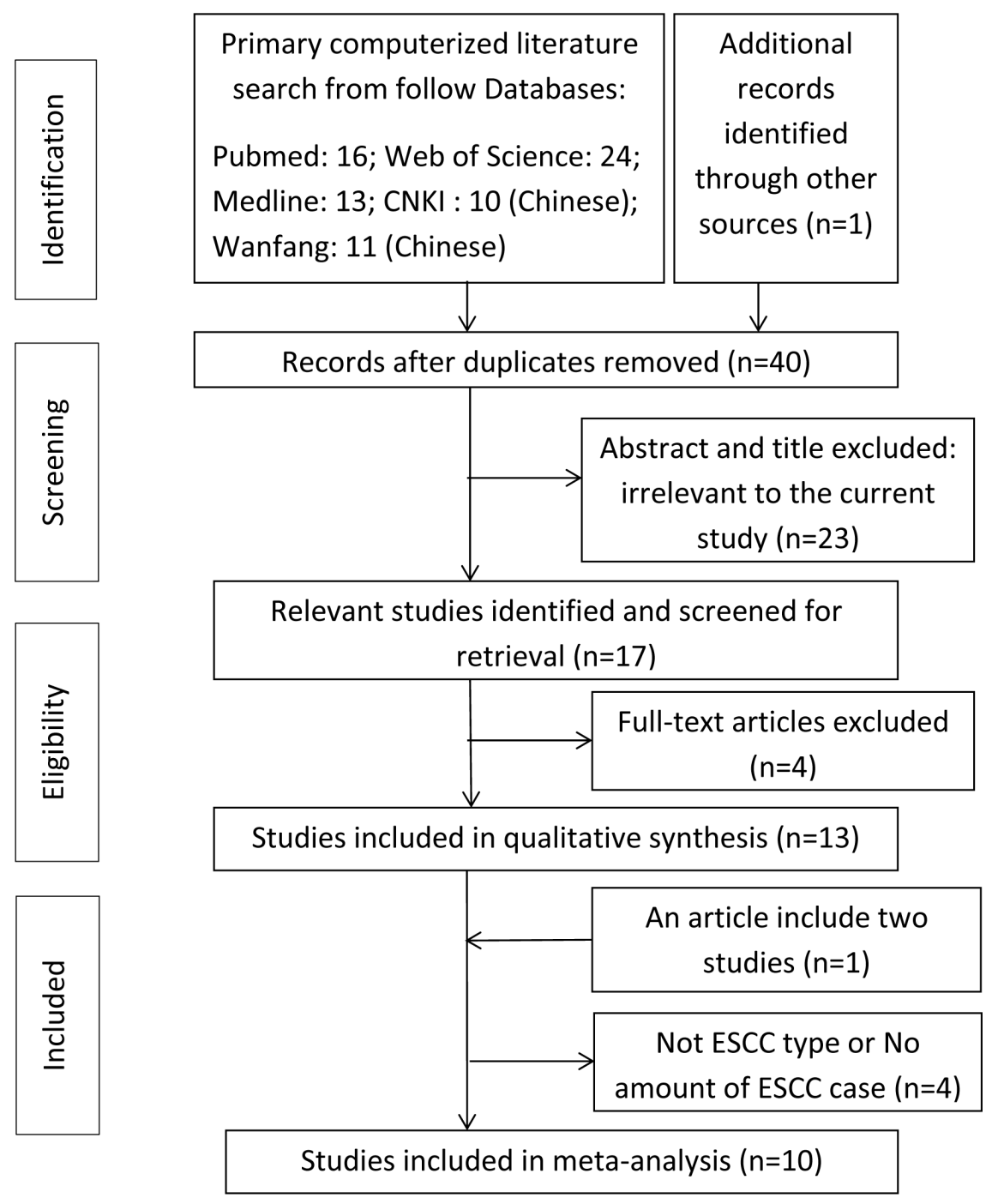

Figure 1. Flow diagram of the study selecting process.

doi:10.1371/journal.pone.0065742.g001

\section{Materials and Methods}

\section{Identification and Eligibility of Relevant Studies}

To identify all published studies that examined the association of hOOG1 Ser326Cys polymorphism with ESCC risks, we conducted a computerized literature search of following databases: PubMed, Web of Science, Medline, China Knowledge Resource Integrated Database (CNKI) and Wanfang database. The key words were as follows: ("esophageal cancer" OR "oesophageal cancer" OR "ESCC"), ("OGG1" OR "hOGG1" OR "8Oxoguanine DNA glycosylase 1"), and ("polymorphism" OR "variation" OR "mutation" OR "SNP"). In the CNKI and Wanfang, corresponding Chinese characters of the keywords were used for searching. References of retrieved articles were also screened. When a study reported results on different subgroups, we treated each subgroup as a single comparison in the metaanalysis. Studies included in this meta-analysis should meet the following criteria: firstly, evaluate the association between hOGG1 Ser326Cys polymorphism and ESCG risk; secondly, use a casecontrol design; thirdly, contain available genotype frequency or genotype frequency can be calculated. Additionally, other relevant studies were identified by hand-searching the references of the eligible articles.

\section{Data Extraction}

Two investigators independently extracted data and reached a consensus on all the items. For each study, the following data were included: the last name of first author, the year of publication, the DNA source of patients (from blood or tumor tissue), ethnicity, the source of controls (population- or hospital-based), genotyping method, numbers of patients and controls, genotypes distribution in each group and published language. We also tested HardyWeinberg equilibrium (HWE) of genotypes distribution in control groups.

\section{Statistical Analysis}

We examined hOGG Ser326Cys genotypes under the dominant (Cys/Cys+Cys/Ser vs. Ser/Ser), recessive (Cys/Cys vs. Cys/ Ser+Ser/Ser), and additive (Cys/Cys vs. Cys/Ser vs. Ser/Ser) models.

The strength of the association between the hOGG1 Ser326Cys polymorphism and ESCC susceptibility was measured by odds 


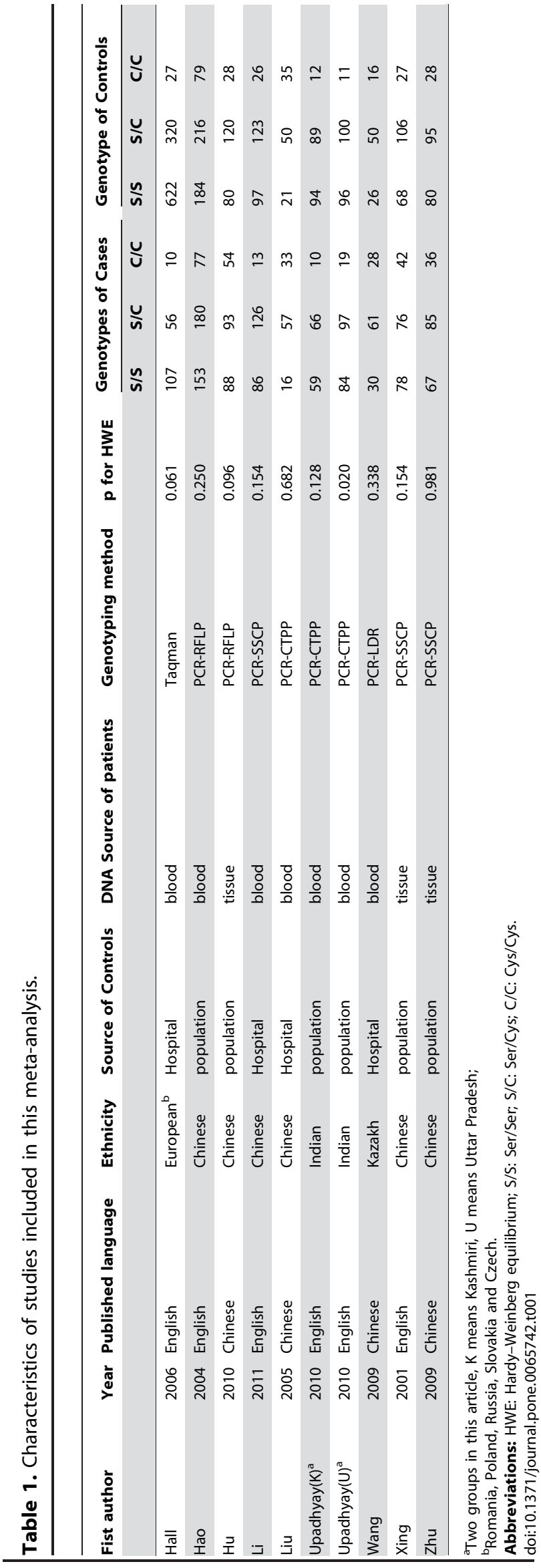

ratios (ORs) with 95\% confidence intervals (CIs). In the dominant and recessive model, the statistical significance of the summary $\mathrm{OR}$ was determined with the $\mathrm{Z}$ test. Heterogeneity assumption was checked by the $Q$ test [13]. If the p-value was greater than 0.1 , a random effect model was used to pool the results. Otherwise, a fixed effects model was then used $[14,15]$. In the additive model, in order to identify the association between the ESCG risk and the number of copies of the 326Cys allele (0, 1 or 2), CochranArmitage trend test was used to calculate $\mathrm{p}$-value for each study $[16,17]$. Greenland and Longnecker's method was used to give the result of overall studies [18].

Stratified analyses were performed by published language (English or Chinese), ethnicity (Asian or Caucasian), source of controls (population- or hospital-based) and the DNA source of patients (blood or tumor tissue).

Meta-regression models were also employed to evaluate the different variance among the individual ORs when heterogeneity was detected. Potential sources of heterogeneity were: published language, ethnicity of the population, source of control and the source of patient DNA.

In order to analyze the deviation of the single study that may denote bias to the overall results, all studies were subjected to a sensitivity analysis. If a study affected the overall result significantly, comprehensive analysis would be taken to find out the cause of heterogeneity. Once found significant heterogeneity, we would like to find out the reason that causes the heterogeneity in the following analysis. Study that deviated from HWE was also removed, for investigating the affection for the overall results. Furthermore, in order to track evidence over time, we also performed a cumulative meta-analysis, in which studies were chronologically ordered by publication year, and pooled ORs were calculated at the end of each year. Funnel plots and Egger's linear regression test were used to provide diagnosis of the potential publication bias [19]. Results were regarded as statistically significant if $\mathrm{P}<0.05$. All statistical tests were performed with Review Manage, version 5.2 and $\mathrm{R}$, version 2.14.1 using two-sided p-values.

\section{Results}

\section{Characteristics of Studies}

There were 40 articles relevant to the search words after removing duplicates records. By reading the titles and abstracts, 23 articles were excluded. Besides, we reviewed the full texts and removed 4 articles because they are not related to our research or not case-control designs. Moreover, we found an article consists of two study groups, so we treated it as two studies in our analysis. Then we checked the type of esophageal cancer and the information about the number of ESCG patients. In this stage, we found that two studies were not related to ESCG, but esophageal adenocarcinoma (EAC) [20,21]. Another two studies contain both types of EC: EAC and ESCG, but we can't get the numbers of ESCG patients even after contacting authors [22,23]. Finally, according to our inclusion criteria, a total of 10 eligible studies, including 6 English articles [8-12] and 4 Chinese studies [24-27], involving 1987 cases and 2926 controls were enrolled in the pooled analyses (Table 1). Among 4 Chinese articles, one of them only has Chinese title and abstract (we have translated the title into English) [24], others have both the title and abstract in English. The flow chart of literature search and study selection was illuminated in Figure 1. These studies were conducted in different populations of various ethnicities: 6 studies of Chinese, 1 study of European, 2 studies of Indian and 1 study of Kazakh. Control sources were from 6 population-based and 4 hospital-based. DNA 
A

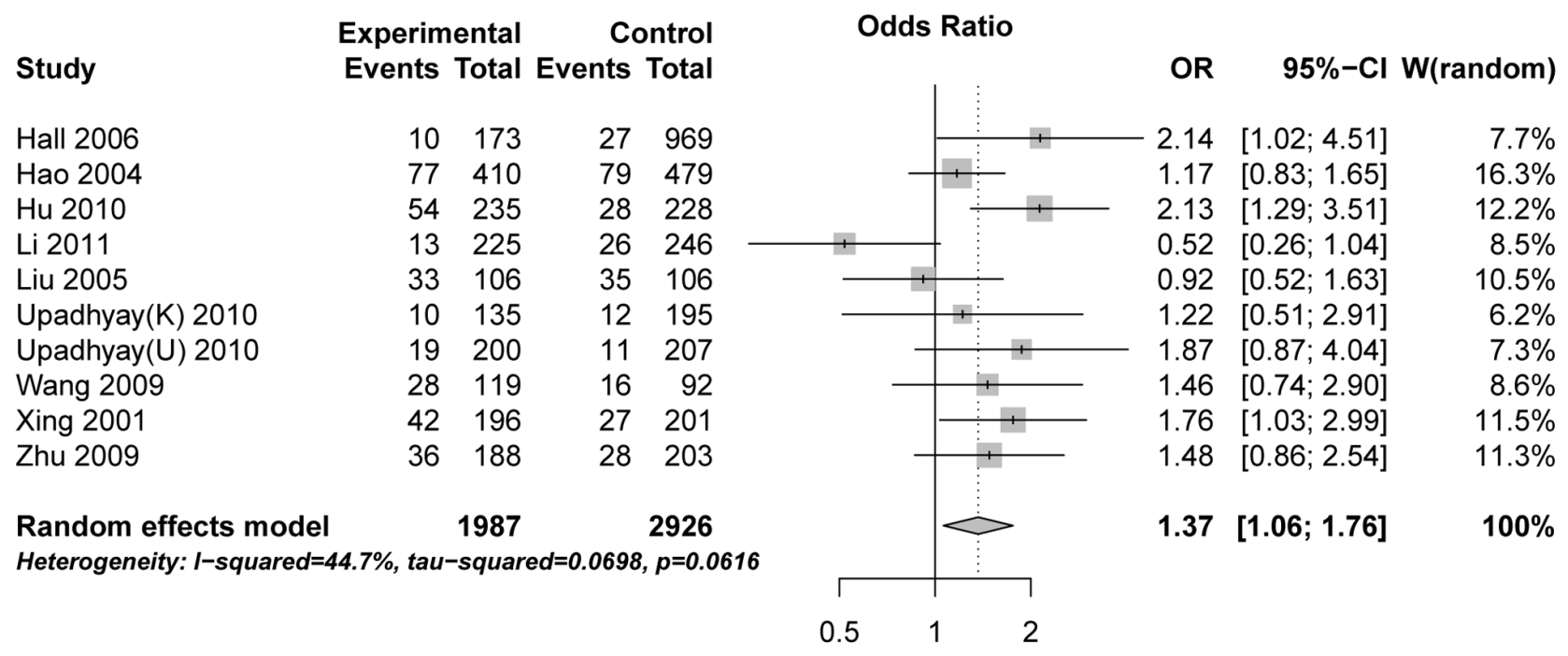

B

\begin{tabular}{lrrrr} 
Study & \multicolumn{2}{c}{$\begin{array}{c}\text { Experimental } \\
\text { Events }\end{array}$} & \multicolumn{2}{c}{$\begin{array}{c}\text { Control } \\
\text { Tovents }\end{array}$} \\
Hall 2006 & 66 & 173 & 347 & 969 \\
Hao 2004 & 257 & 410 & 295 & 479 \\
Hu 2010 & 147 & 235 & 148 & 228 \\
Li 2011 & 139 & 225 & 149 & 246 \\
Liu 2005 & 90 & 106 & 85 & 106 \\
Upadhyay(K) 2010 & 76 & 135 & 101 & 195 \\
Upadhyay(U) 2010 & 116 & 200 & 111 & 207 \\
Wang 2009 & 89 & 119 & 66 & 92 \\
Xing 2001 & 118 & 196 & 133 & 201 \\
Zhu 2009 & 121 & 188 & 123 & 203 \\
& & & & \\
Fixed effect model & \multicolumn{3}{c}{$\mathbf{1 9 8 7}$} \\
Heterogeneity: I-squared $=\mathbf{0 \%}$, tau-squared $=\mathbf{0 ,} \boldsymbol{p}=\mathbf{2 9 . 8 6 9}$
\end{tabular}

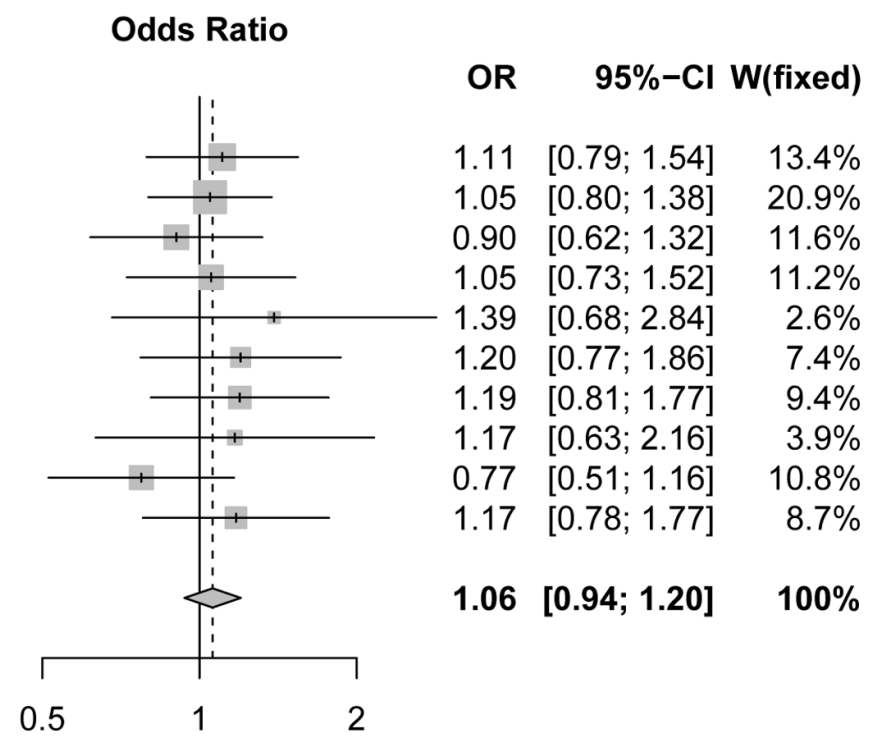

Figure 2. Forest plots of odds ratios (ORs) with $\mathbf{9 5 \%}$ confidence limits (Cls). The center of each square represents the OR. The area of the squares reflects the weight, and the horizontal line indicates the $95 \% \mathrm{Cl}$. (A). recessive model; (B). dominant model. doi:10.1371/journal.pone.0065742.g002

of the patients were extracted from different source, 7 studies from blood and 3 studies from tissue. In addition, genotype distribution of the control group in one study was not consistent with HWE.

\section{Quantitative Synthesis}

We carried out a meta-analysis of the hOGG1 Ser326Cys polymorphism overall, and in subgroups according to ethnic groups, published languages, DNA source of patients and the source of controls. Results of the recessive and dominant model are shown in Table 2 and Table S1, respectively. Forest plots are shown in Figure 2. There was a significant association between the hOGG1 Ser326Cys and ESCC risk in the overall analysis in the recessive model $(\mathrm{OR}=1.37,95 \%$ CI $1.06-1.76, \quad \mathrm{P}=0.02)$. However, the result was not significant under a dominant model $(\mathrm{OR}=1.06,95 \% \mathrm{CI}: 0.94-1.20 ; \mathrm{P}=0.36)$. There was significant heterogeneity between studies under the recessive model $(\mathrm{P}=0.06$; $\left.\mathrm{I}^{2}=45 \%\right)$ but not the dominant model $\left(\mathrm{P}=0.87 ; \mathrm{I}^{2}=0 \%\right)$. In subgroup analysis, the hOGGl Cys/Cys polymorphism was significantly associated with the risk of ESCC in the recessive model performed by ethnicity, published language, DNA source of patients and the source of control groups. We found that Cys/Cys variants in subgroups of Chinese language, Caucasian, controls of population and tissue increased ESCG risks, with the ORs of 1.49 (95\% CI 1.12-1.96, $\mathrm{p}=0.006), 1.64(95 \%$ CI 1.12-2.40, $\mathrm{p}=0.01)$, $1.50(1.22-1.85, \mathrm{p}=0.0001), 1.79(1.33-2.42, \mathrm{p}=0.001)$, respectively. However, no significant result was observed in the dominant model in any subgroup. In the additive model, pairwise comparison of genotypes and p-value of the Cochran-Armitage test for each study are shown in Table S2. Overall and subgroup results are shown in Table S3. Positive correlation was found between the number of Cys allele and the risk of ESCC $(\mathrm{PCC}=0.109, \mathrm{SE}=0.046, \mathrm{p}=0.02)$. In subgroup analysis, the risk of ESCG is positively correlated with the number of Cys allele in Caucasian subgroup $(\mathrm{PCC}=0.19, \mathrm{SE}=0.084, \mathrm{p}=0.02)$ and 
Table 2. Quantitative analyses and the test of heterogeneity of the hOGG1 Ser326Cys polymorphism on the ESCC risk in a recessive model.

\begin{tabular}{|c|c|c|c|c|c|c|c|}
\hline & \multicolumn{4}{|l|}{ Q-test } & \multirow[b]{2}{*}{ sample size ${ }^{a}$} & \multirow[b]{2}{*}{ OR(95\%Cl) } & \multirow[b]{2}{*}{ p-Value } \\
\hline & chi-squared & df & p-Value & $I^{2}(\%)$ & & & \\
\hline Overall & 16.26 & 9 & 0.06 & 45 & 4913 & $1.37(1.06,1.76)$ & $0.02^{\mathrm{b}}$ \\
\hline \multicolumn{8}{|l|}{ Ethnic group } \\
\hline Asian & 13.75 & 5 & 0.02 & 63.6 & 2823 & $1.25(0.88,1.77)$ & $0.21^{\mathrm{b}}$ \\
\hline Caucasian & 1.16 & 3 & 0.76 & 0 & 2090 & $1.64(1.12,2.40)$ & 0.01 \\
\hline \multicolumn{8}{|l|}{ Published language } \\
\hline English language & 10.97 & 5 & 0.05 & 54.4 & 3636 & $1.31(0.90,1.90)$ & $0.16^{\mathrm{b}}$ \\
\hline Chinese language & 4.7 & 3 & 0.2 & 36.1 & 1277 & $1.49(1.12,1.96)$ & 0.006 \\
\hline \multicolumn{8}{|l|}{ Source of controls } \\
\hline Population & 4.75 & 5 & 0.45 & 0 & 2877 & $1.50(1.22,1.85)$ & 0.0001 \\
\hline Hospital & 8.64 & 3 & 0.03 & 65.3 & 2036 & $1.09(0.62,1.93)$ & $0.77^{b}$ \\
\hline \multicolumn{8}{|l|}{ DNA source } \\
\hline blood & 10.37 & 6 & 0.11 & 42.2 & 3662 & $1.15(0.93,1.44)$ & 0.2 \\
\hline tissue & 0.95 & 2 & 0.62 & 0 & 1251 & $1.79(1.33,2.42)$ & 0.0001 \\
\hline
\end{tabular}

aSample size equals the total number of controls and cases;

${ }^{\mathrm{b}}$ Caculated by random model, otherwise by fixed model.

Abbreviations: OR: odds ratio; $\mathrm{Cl}$ : confidence intervals.

doi:10.1371/journal.pone.0065742.t002

population subgroup $(\mathrm{PCC}=0.12, \mathrm{SE}=0.055, \mathrm{p}=0.03)$. No heterogeneity was found between studies $\left(\mathrm{P}=0.94 ; \mathrm{I}^{2}=0 \%\right)$.

Meta-regression analysis under the recessive model was performed in order to find out potential sources of heterogeneity. Empty regression was firstly run to estimate the baseline value for $\operatorname{tau}^{2}(0.070)$. In the meta-regression analysis, the model including DNA source of patients reduced the $\operatorname{tau}^{2}$ value to 0.014 (beta coefficient $=1.993,95 \%$ CI: $0.007-0.832 ; \mathrm{P}=0.046)$, suggesting DNA source of patients was a significant source of heterogeneity in the recessive model.

Study

Omitting Hall 2006

Omitting Hao 2004

Omitting Hu 2010

Omitting Li 2011

Omitting Liu 2005

Omitting Upadhyay $(\mathrm{K}) 2010$

Omitting Upadhyay(U) 2010

Omitting Wang 2009

Omitting Xing 2001

Omitting Zhu 2009

Random effects model
Odds Ratio

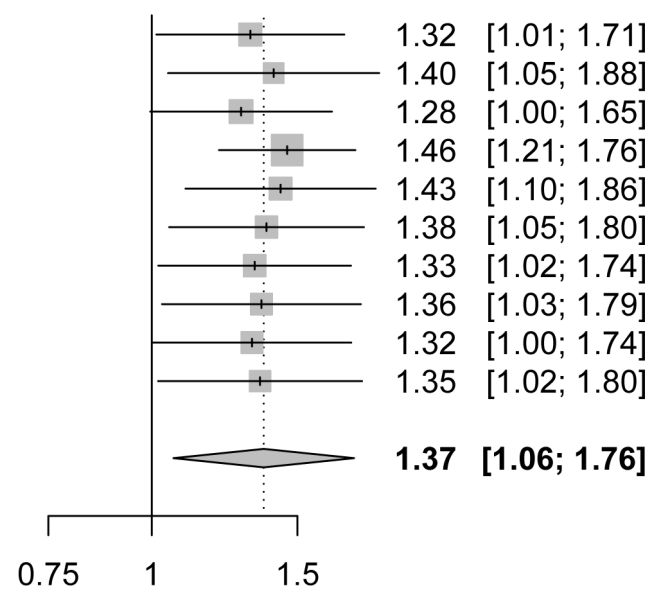

\section{OR $\quad 95 \%-\mathrm{Cl}$}

$.32[1.01 ; 1.71]$

$40 \quad[1.05 ; 1.88]$

$.28[1.00 ; 1.65]$

$.46 \quad[1.21 ; 1.76]$

$43[1.10 ; 1.86]$

$38 \quad[1.05 ; 1.80]$

$1.33[1.02 ; 1.74]$

$1.36 \quad[1.03 ; 1.79]$

$.32[1.00 ; 1.74]$

$35[1.02 ; 1.80]$

$1.37[1.06 ; 1.76]$

\section{$0.75 \quad 1 \quad 1.5$}

Sensitivity Analyses and Adjusted Results of Metaanalysis

Since significant heterogeneity across studies was observed for the recessive model, we conducted a sensitivity analysis to assess the influence of each individual study on the pooled OR and the heterogeneity by sequentially removing the individual study. Results are summarized in Table 3 . The forest plot of sensitivity analysis in a random model is shown in Figure 3. We can see that removing the HWE-deviation study (Upadhyay 2010 "UP" population) did not affect the result significantly. We also found that the study conducted by $\mathrm{Li}$ et al. (2011) influents the overall pooled estimates and the heterogeneity most. We compared this study to other studies carefully and finally found a significant

Figure 3. Results of odds ratios (ORs) with $\mathbf{9 5 \%}$ confidence limits (CIs) in sensitivity analysis. Results were computed by omitting each study. Random-effects estimates were used. Each OR means the result when remove the corresponding study. doi:10.1371/journal.pone.0065742.g003 


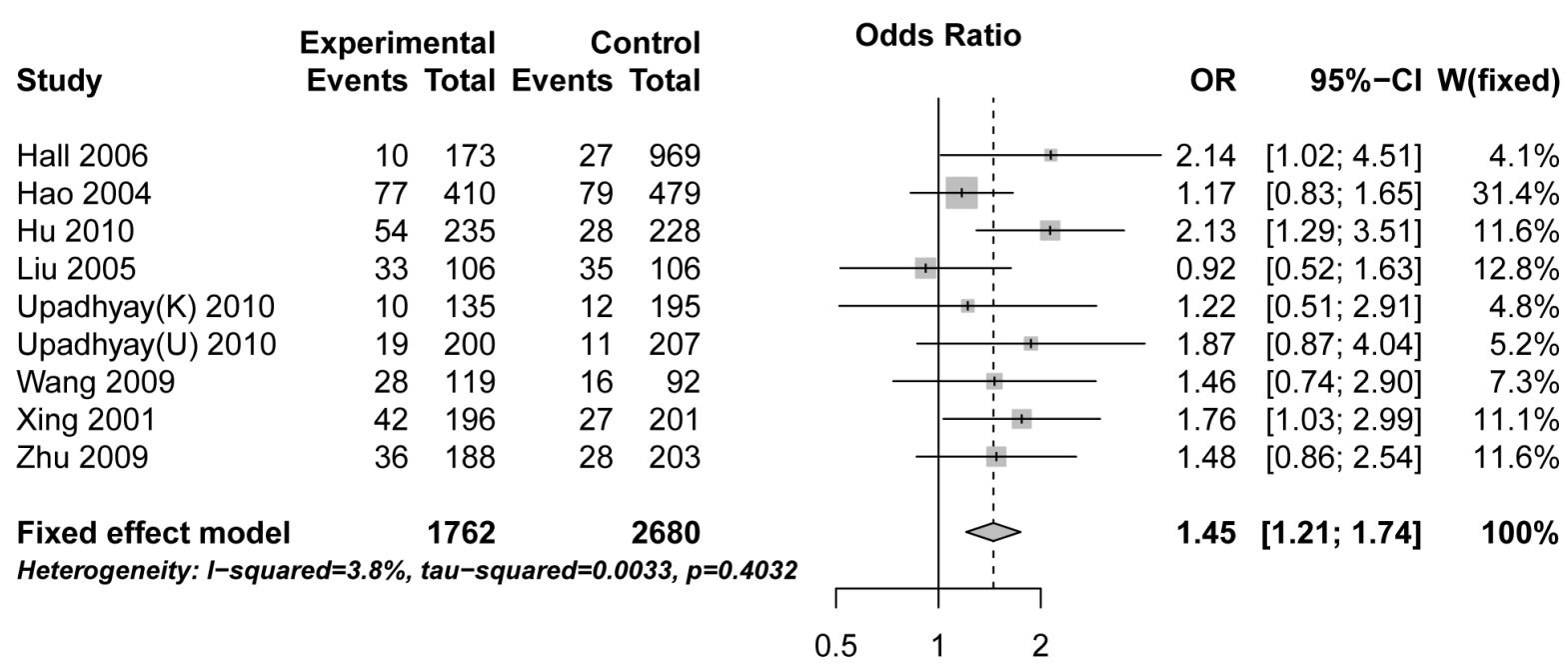

Figure 4. Forest plots result without the study of $\mathbf{L i}$ et al. The center of each square represents the OR, the area of the square is the weight used in the meta-analysis, and the horizontal line indicates the $95 \% \mathrm{Cl}$. doi:10.1371/journal.pone.0065742.g004

difference of sex and age between the case and the control in this study. The sex and age information for each study is listed in Table 4. Cases and controls matched by sex and age in most studies. However, in the study of Li et al. (2011), we observed a significant difference between the case and control both by sex $(\mathrm{p}<0.001)$ and age $(\mathrm{p}<0.001)$. Besides, sex ratios between the case and control group in the study of Hall et al. (2006) were not matched well $(\mathrm{p}<0.001)$. However, in the sensitivity analysis, this article did not influent the heterogeneity very much. This time, after omitting the $\mathrm{Li}$ et al. (2011), the overall heterogeneity between studies was not significant $\left(\mathrm{P}=0.40, \mathrm{I}^{2}=3.8\right)$. These results proved that the study of $\mathrm{Li}$ et al. (2011) has a significant heterogeneity from other studies. After removing the study of $\mathrm{Li}$ et al. (2011), overall and subgroups ORs in 9 studies were calculated again in the recessive model. Results are summarized in Table S4. No significant heterogeneity was found in any subgroups. Results were in fixed models and the overall
$\mathrm{OR}=1.45$ (95\% CI 1.21-1.74, $\mathrm{p}<0.0001$, Figure 4). Comparing the former subgroups analysis, results had some changes. As shown in Table S4, all results of subgroups reached significant levels. Then, a new sensitivity analysis was performed on the remained 9 studies. Before and after deleting each study, no significant heterogeneity between the remaining studies was found (Table S5). Forest plot of sensitivity analysis in the fixed model is shown in Figure S1. The outcomes were all similar after removing each study. These results suggest that no individual study significantly affected the overall OR in the new meta-analysis.

\section{Cumulative Meta-analysis and Publication Bias}

Cumulative meta-analysis of hOGG1 ser326cys with ESCG was conducted via the assortment of studies by publication time. Inclinations towards significant association were evident over time in the recessive model (Figure 5), but not in the dominant model

Table 3. Sensitivity analysis of 10 studies (recessive model).

\begin{tabular}{|c|c|c|c|c|c|c|c|}
\hline \multirow[b]{2}{*}{ Study Omitted } & \multicolumn{2}{|l|}{ Fix Model } & \multicolumn{2}{|l|}{ Random model } & \multicolumn{3}{|c|}{ heterogeneity } \\
\hline & OR(95\%-CI) & p-Value & OR(95\%-CI) & p-Value & $\operatorname{tau}^{2}$ & $I^{2}(\%)$ & p-Value \\
\hline None & $1.348(1.130,1.608)$ & 0.001 & $1.366(1.062,1.756)$ & 0.015 & 0.070 & 44.7 & 0.06 \\
\hline Hall 2006 & $1.318(1.099,1.580)$ & 0.003 & $1.316(1.013,1.709)$ & 0.040 & 0.070 & 45.7 & 0.06 \\
\hline Hao 2004 & $1.416(1.153,1.740)$ & 0.001 & $1.404(1.047,1.884)$ & 0.024 & 0.094 & 47.8 & 0.05 \\
\hline Hu 2010 & $1.257(1.040,1.520)$ & 0.018 & $1.282(0.996,1.651)$ & 0.054 & 0.053 & 36.8 & 0.12 \\
\hline Li 2011 & $1.451(1.207,1.744)$ & $<0.0001$ & $1.458(1.206,1.763)$ & $<0.0001$ & 0.003 & 3.8 & 0.40 \\
\hline Liu 2005 & $1.403(1.165,1.689)$ & $<0.001$ & $1.431(1.099,1.865)$ & 0.008 & 0.069 & 44.1 & 0.07 \\
\hline Upadhyay $2010^{a}$ & $1.354(1.130,1.621)$ & 0.001 & $1.377(1.050,1.805)$ & 0.021 & 0.083 & 50.6 & 0.04 \\
\hline Upadhyay $2010^{\mathrm{b}}$ & $1.322(1.103,1.586)$ & 0.003 & $1.332(1.019,1.741)$ & 0.036 & 0.078 & 48.6 & 0.05 \\
\hline Wang 2009 & $1.340(1.116,1.609)$ & 0.002 & $1.357(1.029,1.790)$ & 0.031 & 0.086 & 50.7 & 0.04 \\
\hline Xing 2001 & $1.303(1.080,1.572)$ & 0.006 & $1.322(1.003,1.743)$ & 0.048 & 0.080 & 47.5 & 0.05 \\
\hline Zhu 2009 & $1.333(1.105,1.607)$ & 0.003 & $1.352(1.018,1.796)$ & 0.037 & 0.090 & 50.5 & 0.04 \\
\hline
\end{tabular}


Table 4. Sex and age information extracted from original articles.

\begin{tabular}{|c|c|c|c|c|c|c|c|c|c|}
\hline \multirow[t]{3}{*}{ First author } & \multirow[t]{3}{*}{ Year } & \multicolumn{5}{|l|}{ Sex } & \multicolumn{3}{|l|}{ Age } \\
\hline & & \multicolumn{2}{|l|}{ Case } & \multicolumn{2}{|c|}{ Control } & \multirow[t]{2}{*}{ Result } & \multirow{2}{*}{ Case } & \multirow[t]{2}{*}{ Control } & \multirow{2}{*}{ Result } \\
\hline & & Male & Female & Male & Female & & & & \\
\hline \multirow[t]{7}{*}{ Hall } & 2006 & 713 & 98 & 831 & 252 & $\mathrm{p}<0.001$ & $<50: 140$ & $<50: 203$ & $p=0.095$ \\
\hline & & & & & & & $50-54: 148$ & $50-54: 170$ & \\
\hline & & & & & & & $55-59: 155$ & $55-59: 178$ & \\
\hline & & & & & & & $60-64: 142$ & $60-64: 174$ & \\
\hline & & & & & & & 65-69: 106 & 65-69: 162 & \\
\hline & & & & & & & 70-74: 99 & 70-74: 149 & \\
\hline & & & & & & & $\geq 75: 21$ & $\geq 75: 47$ & \\
\hline Hao & 2004 & N.A. & N.A. & N.A. & N.A. & match $^{a}$ & N.A. & N.A. & match $^{\mathrm{a}}$ \\
\hline $\mathrm{Hu}$ & 2010 & 171 & 64 & 167 & 61 & $p=0.907$ & $61.60 \pm 10.06$ & $61.57 \pm 10.17$ & $p=0.974$ \\
\hline \multirow[t]{3}{*}{ Li } & 2011 & 162 & 64 & 125 & 121 & $p<0.001$ & $<50: 38$ & $<50: 142$ & $p<0.001$ \\
\hline & & & & & & & $50-60: 102$ & $50-60: 66$ & \\
\hline & & & & & & & $\geq 60: 86$ & $\geq 60: 38$ & \\
\hline Liu & 2005 & 52 & 54 & 52 & 54 & $p=1.000$ & N.A. & N.A. & match $^{a}$ \\
\hline \multirow[t]{2}{*}{ Upadhyay } & $2010^{b}$ & 92 & 43 & 139 & 56 & $p=0.541$ & $60.38 \pm 8.40$ & $57.98 \pm 12.66$ & $p=0.055$ \\
\hline & $2010^{c}$ & 147 & 53 & 152 & 55 & $p=0.987$ & $56.52 \pm 12.09$ & $55.41 \pm 10.35$ & $p=0.300$ \\
\hline Wang & 2009 & 80 & 52 & 83 & 50 & $p=0.763$ & N.A. & N.A. & match $^{\mathrm{a}}$ \\
\hline Xing & 2001 & 140 & 56 & 143 & 58 & $p=0.950$ & $55.8 \pm 9.0$ & $55.5 \pm 8.6$ & $p=0.734$ \\
\hline Zhu & 2009 & 105 & 83 & 126 & 77 & $p=0.212$ & 61.03 & 60.77 & $p=0.061^{a}$ \\
\hline
\end{tabular}

a Described in the original article;

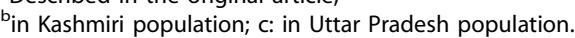

Abbreviations: N.A: Not Available.

doi:10.1371/journal.pone.0065742.t004

(Figure S2). These results suggest that the precision of the estimates was progressively boosted by continually adding more samples.

Funnel plot and Egger's test were performed to assess publication bias. The shapes of the funnel plots indicate that there was no obvious asymmetry. And the Egger's test also shows no publication bias (recessive: $\mathrm{t}=0.27, \mathrm{p}=0.79$; dominant: $\mathrm{t}=0.91, \mathrm{p}=0.39 ;$ Figure 6).

\section{Study}

Adding Xing $2001(\mathrm{k}=1)$

Adding Hao 2004 ( $\mathrm{k}=2$ )

Adding Liu $2005(\mathrm{k}=3)$

Adding Hall $2006(\mathrm{k}=4)$

Adding Wang $2009(\mathrm{k}=5)$

Adding Zhu 2009 ( $k=6)$

Adding $\mathrm{Hu} 2010$ ( $\mathrm{k}=7$ )

Adding Upadhyay(K) 2010 (k=8)

Adding Upadhyay(U) $2010(\mathrm{k}=9)$

Adding Li $2011(\mathrm{k}=10)$

\section{Random effects model}

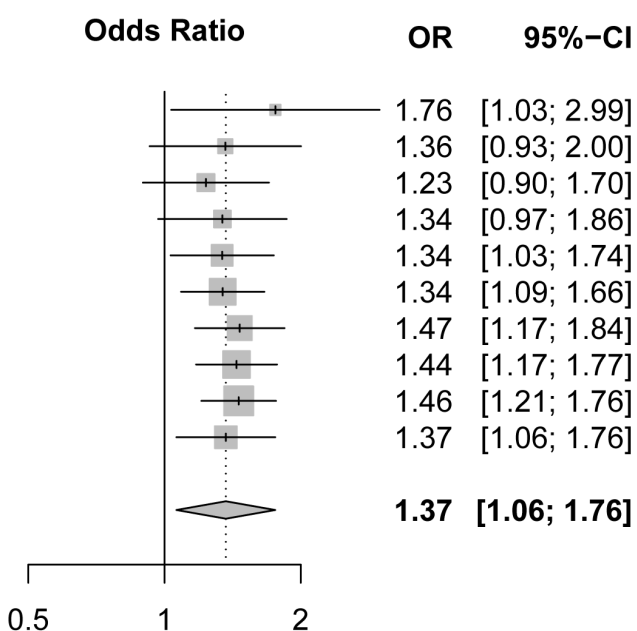

Figure 5. Forest plots of results of cumulative meta-analysis by published year in the recessive model. Pooled odds ratios (ORs) with $95 \%$ confidence limits $(\mathrm{Cls})$ at the end of each information step were shown. doi:10.1371/journal.pone.0065742.g005

\section{Discussion}

Oxidative DNA damage occurs in a cell when the production of reactive oxygen species (ROS) exceeds the cell's antioxidantdefense capacity, leading to cell apoptosis and producing mutations in the DNA $[28,29]$. Among many factors of oxidative DNA damage, 8-hydroy-2-deoxyguanine $(8-\mathrm{OHdG})$ is one of the most abundant oxidative products of highly mutagenic because of 
A

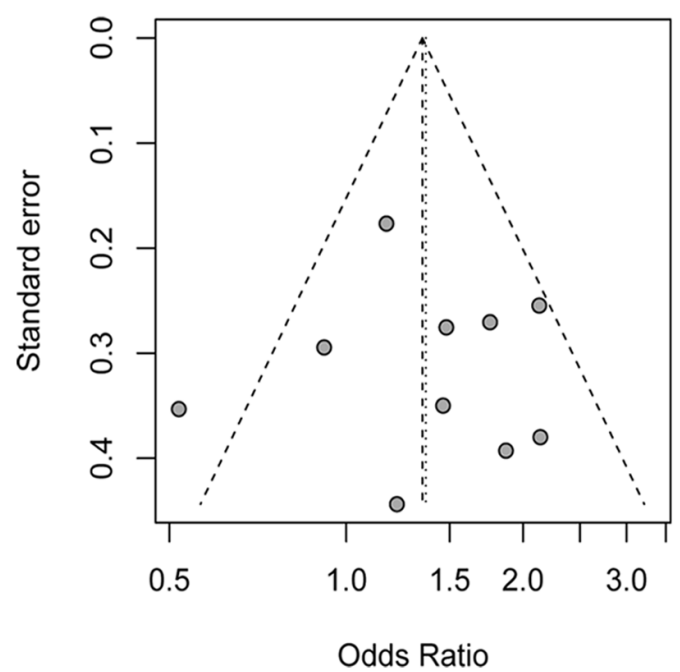

B

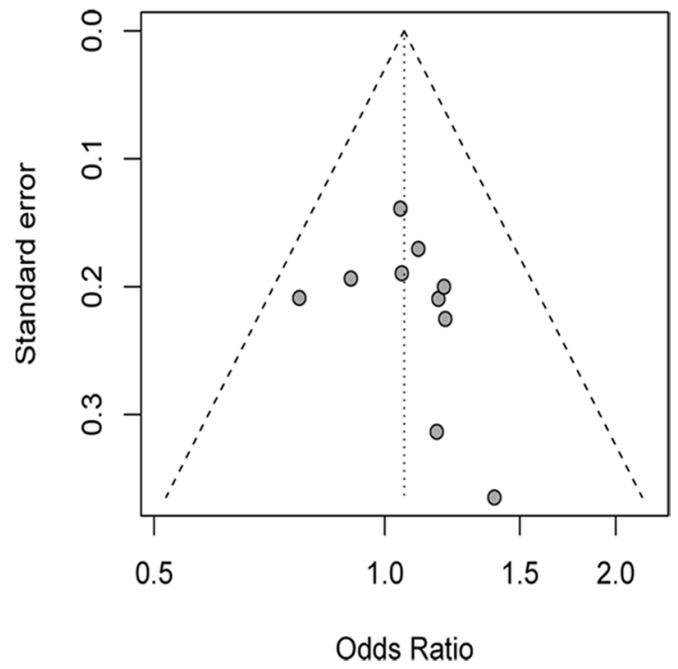

Figure 6. Begg's funnel plot with $\mathbf{9 5 \%}$ confidence limits (Cls) of publication bias test. (A). recessive model; (B). dominant model. doi:10.1371/journal.pone.0065742.g006

its propensities to mispair with adenine during DNA replication and to cause ultimately GC to TA mutation [30].

Many studies have reported that the hOGGl gene could remove $8-\mathrm{OHdG}$ from DNA by base excision repair (BER) pathway [31,32]. If hOGGl was dysfunctional, the damage could be left unrepaired, leading to mutations or carcinogenesis. A polymorphism of the hOGGl has been identified, which caused an amino substitution from serine to cysteine in a codon 326. A number of studies have suggested that this variation may be associated with increased risks of several kinds of cancer. Several of them have focused on the association between the hOGGl Ser326Cys polymorphism and esophageal cancer risk, but the results were inconclusive. Hence, this meta-analysis was needed to provide a quantitative approach for combining the different results.

The present meta-analysis includes 1987 esophageal cancer cases and 2926 controls. As far as we know, this is the first metaanalysis carried out with the aim of investigating the relationship between hOGG1 Ser326Cys polymorphism and ESCG risks. Results were significant in the recessive and additive model. Comparing with the Ser/Ser and Ser/Cys genotype, Cys/Cys genotype might contribute to increase the risk of ESCC. And the ESCG risk is positively correlated with the number of Cys allele.

When taking the sensitivity analyses, we found an article that inference the overall result very much and have heterogeneity to other studies. When we remove this study, the heterogeneity disappeared and the results became more stable. By comparing this article to others, we finally found the sex rate and age in the case group were significantly different from those in the control group. Some studies have found that ESCC most often occurs in men, and more often seen in aged 50-70 [33,34]. These results suggest that when designing a case-control study of genetic polymorphism and cancer risk, investigator should match case and control well in sex rate and age, in order to give a reliable conclusion.

In many previous studies of meta-analysis, HWE-deviation studies were often ignored in the pooled estimate. However, a researcher argued that studies that appear to deviate from HWE should not be excluded unless there are other grounds for doubting the quality of the study [35]. In the sensitivity analysis of our research, no significant change of the OR was found when we remove the HWE-deviation study (Upadhyay 2010 "UP" Population). This result was consistent with that article. Therefore this study was included in our research.

In subgroup analysis by esophageal cancer types, we found that the hOGG1 Ser326Cys polymorphism was significantly associated with ESCG in the Caucasian population. However, the number of studies in this subgroup was a little small $(n=4)$, which may reduce the reliability of the results. Interestingly, we found a significant association between hOGG1 Ser326Cys polymorphism and ESCG in population controls group rather than controls based on the hospital. This finding suggests that Cys/Cys carrier may also cause other diseases risks in addition to ESCC.

In conclusion, our meta-analysis suggests that the hOGG1 Ser326Cys polymorphism is associated with esophageal cancer susceptibility. Gys/Cys carriers have more risk on ESCC rather than Ser/Ser and Ser/Cys carriers. Each copy of Cys modifies the risk of ESCG in an additive form. It is the homozygous Cys/Cys have a higher risk than heterozygous Cys/Ser. Moreover, in order to get a more reliable result, some factors such as gender and age should be matched in a case-control design study.

Several limitations of this meta-analysis should be addressed. First, we abandoned 2 studies when we read the full text of articles because they are mixed cancer types (EAC and ESCG); but we cannot get the numbers of ESCG patients from studies or authors. This limited the scale of the data to the meta-analysis. Second, genotypes errors may also influence the results, because the quality control of genotypic was not well documented in some studies. Third, several studies had a relatively small sample size.

In order to provide a more precise estimation, further research is necessary to use standardized unbiased homogenous cancer patients and well-matched controls to investigate the combined effects. That would lead to a better, comprehensive understanding of the association between hOGG1 Ser326Cys polymorphism and esophageal cancer risk. 


\section{Supporting Information}

Figure S1 Sensitivity analysis without the study of Li et al. Results were computed by omitting each study. Fixedeffects estimates were used. Each OR means the result when remove the corresponding study.

(PNG)

Figure S2 Forest plots of cumulative analysis in the dominant model. Pooled odds ratios (ORs) with 95\% confidence limits (CIs) at the end of each information step were shown.

(PNG)

Table S1 Overall and subgroup results in the dominant models.

(DOC)

Table S2 Pairwise comparison and $P$-valule in the additive models.

\section{References}

1. Umar SB, Fleischer DE (2008) Esophageal cancer: epidemiology, pathogenesis and prevention. Nat Clin Pract Gastroenterol Hepatol 5: 517-526.

2. He J, Gu D, Wu X, Reynolds K, Duan X, et al. (2005) Major causes of death among men and women in China. N Engl J Med 353: 1124-1134.

3. Mohrenweiser HW, Wilson DM, Jones IM (2003) Challenges and complexities in estimating both the functional impact and the disease risk associated with the extensive genetic variation in human DNA repair genes. Mutat Res 526: 93125.

4. Dherin C, Radicella JP, Dizdaroglu M, Boiteux S (1999) Excision of oxidatively damaged DNA bases by the human alpha-hOggl protein and the polymorphic alpha-hOgg1(Ser326Cys) protein which is frequently found in human populations. Nucleic Acids Res 27: 4001-4007.

5. Audebert M, Radicella JP, Dizdaroglu M (2000) Effect of single mutations in the OGG1 gene found in human tumors on the substrate specificity of the Ogg1 protein. Nucleic Acids Res 28: 2672-2678.

6. Nishioka K, Ohtsubo T, Oda H, Fujiwara T, Kang D, et al. (1999) Expression and differential intracellular localization of two major forms of human 8oxoguanine DNA glycosylase encoded by alternatively spliced OGG1 mRNAs. Mol Biol Cell 10: 1637-1652.

7. Kohno T, Shinmura K, Tosaka M, Tani M, Kim SR, et al. (1998) Genetic polymorphisms and alternative splicing of the hOGG1 gene, that is involved in the repair of 8-hydroxyguanine in damaged DNA. Oncogene 16: 3219-3225.

8. Hall J, Hashibe M, Boffetta P, Gaborieau V, Moullan N, et al. (2007) The association of sequence variants in DNA repair and cell cycle genes with cancers of the upper aerodigestive tract. Carcinogenesis 28: 665-671.

9. Hao B, Wang H, Zhou K, Li Y, Chen X, et al. (2004) Identification of genetic variants in base excision repair pathway and their associations with risk of esophageal squamous cell carcinoma. Cancer Res 64: 4378-4384.

10. Li QD, Li H, Wang MS, Diao TY, Zhou ZY, et al. (2011) Multi-susceptibility genes associated with the risk of the development stages of esophageal squamous cell cancer in Feicheng County. BMC Gastroenterol 11: 74.

11. Upadhyay R, Malik MA, Zargar SA, Mittal B (2010) OGG1 Ser326Cys polymorphism and susceptibility to esophageal cancer in low and high at-risk populations of northern India. J Gastrointest Cancer 41: 110-115.

12. Xing DY, Tan W, Song N, Lin DX (2001) Ser326Cys polymorphism in hOGG1 gene and risk of esophageal cancer in a Chinese population. Int J Cancer 95: $140-143$.

13. Handoll HH (2006) Systematic reviews on rehabilitation interventions. Arch Phys Med Rehabil 87: 875.

14. Mantel N, Haenszel W (1959) Statistical aspects of the analysis of data from retrospective studies of disease. J Natl Cancer Inst 22: 719-748.

15. DerSimonian R, Laird N (1986) Meta-analysis in clinical trials. Control Clin Trials 7: $177-188$

16. Cochran WG (1954) SOME METHODS FOR STRENGTHENING THE COMMON X2 TESTS. Biometrics 10: 417-451.

17. Armitage P (1955) TESTS FOR LINEAR TRENDS IN PROPORTIONS AND FREOUENCIES. Biometrics 11: 375-386.

18. Greenland S, Longnecker MP (1992) Methods for trend estimation from summarized dose-response data, with applications to meta-analysis. Am J Epidemiol 135: 1301-1309.

19. Egger M, Davey Smith G, Schneider M, Minder G (1997) Bias in meta-analysis detected by a simple, graphical test. BMJ 315: 629-634.

20. Ferguson HR, Wild CP, Anderson LA, Murphy SJ, Johnston BT, et al. (2008) No association between hOGG1, XRCG1, and XPD polymorphisms and risk of reflux esophagitis, Barrett's esophagus, or esophageal adenocarcinoma: results
(DOC)

Table S3 Overall and subgroup analysis in the additive models.

(DOC)

Table S4 Results after removing the study of $\mathrm{Li}$ et al. in the recessive models.

(DOC)

Table S5 Sensitivity analysis of 9 studies in the recessive model.

(DOC)

\section{Author Contributions}

Conceived and designed the experiments: ST MW WW. Performed the experiments: JJZ JSZ. Analyzed the data: JJZ JSZ PZ. Contributed reagents/materials/analysis tools: JJZ JSZ PZ WW. Wrote the paper: JJZ JSZ.

from the factors influencing the Barrett's adenocarcinoma relationship casecontrol study. Cancer Epidemiol Biomarkers Prev 17: 736-739.

21. Tse D, Zhai R, Zhou W, Heist RS, Asomaning K, et al. (2008) Polymorphisms of the NER pathway genes, ERCC1 and XPD are associated with esophageal adenocarcinoma risk. Cancer Causes Control 19: 1077-1083.

22. Lagadu S, Lechevrel M, Sichel F, Breton J, Pottier D, et al. (2010) 8-oxo-7,8dihydro-2'-deoxyguanosine as a biomarker of oxidative damage in oesophageal cancer patients: lack of association with antioxidant vitamins and polymorphism of hOGG1 and GST. J Exp Clin Cancer Res 29: 157.

23. Gao C, Sugimura H, Takezaki T, Wu J, Li Z, et al. (2001) hOGG1 Genotypes, Life Style and the Risk of Esophageal and Stomach Cancers. Bulletin of Chinese Cancer 10: 3. CNKI database. Available: http://en.cnki.com.cn/Article_en/ CJFDTOTAL-ZHLU200109001.htm. Accessed 2013 May 8. [Article in Chinese].

24. Hu H, Wu J, Jin Y, Zhang L, Gao G (2010) hOGG1 genotype with susceptibility to esophageal cancer in Northern Henan regions. Shandong Medical Journal 50: 2. Wanfang database. Available: http://d.wanfangdata.com.cn/Periodical shandyy201032026.aspx. Accessed 2013 May 8. [Article in Chinese].

25. Liu R, Yin L, Pu Y, Liu Y, Hu X, et al. (2005) Relationship between human 8hydroxyguanine glycosylase Ser326Cys gene polymorphism and esophageal cancer. China Public Health 21: 3. CNKI database. Available: http://en.cnki. com.cn/Article_en/CJFDTOTAL-ZGGW200512011.htm. Accessed 2013 May 8. [Article in Chinese]

26. Wang Y, Zhang C, Chen Y, Deng Y, Ma Y, et al. (2009) Relationship between hOGGl gene polymorphism and the susceptibility of esophageal cancer in Kazakh nationality. Journal of Xinjiang Medical University 32: 4. CNKI database. Available: http://en.cnki.com.cn/Article_en/CJFDTOTALXJYY200905008.htm. Accessed 2013 May 8. [Article in Chinese].

27. Zhu X, Zhang H, Du H, Hao O, Wu X, et al. (2009) Association of hOGG1 Polymorphism Ser326Cys between the Susceptibility of Esophageal Cancer and Its Clinicopathological Characteristics. Journal of Environmental \& Occupational Medicine 26: 4. CNKI database. Available: http://en.cnki.com.cn/ Article_en/CJFDTOTAL-LDYX200902009.htm. Accessed 2013 May 8. [Article in Chinese].

28. Roy D, Liehr JG (1999) Estrogen, DNA damage and mutations. Mutat Res 424: $107-115$.

29. Yoshie Y, Ohshima H (1998) Synergistic induction of DNA strand breakage by catechol-estrogen and nitric oxide: implications for hormonal carcinogenesis. Free Radic Biol Med 24: 341-348.

30. Shibutani S, Takeshita M, Grollman AP (1991) Insertion of specific bases during DNA synthesis past the oxidation-damaged base 8-oxodG. Nature 349: 431434.

31. Boiteux S, Radicella JP (1999) Base excision repair of 8-hydroxyguanine protects DNA from endogenous oxidative stress. Biochimie 81: 59-67.

32. Boiteux S, Radicella JP (2000) The human OGG1 gene: structure, functions, and its implication in the process of carcinogenesis. Arch Biochem Biophys 377: $1-8$.

33. Bahmanyar S, Zendehdel K, Nyren O, Ye W (2007) Risk of oesophageal cancer by histology among patients hospitalised for gastroduodenal ulcers. Gut 56: 464 468.

34. Johnell O, Kanis JA (2004) An estimate of the worldwide prevalence, mortality and disability associated with hip fracture. Osteoporos Int 15: 897-902.

35. Minelli C, Thompson JR, Abrams KR, Thakkinstian A, Attia J (2008) How should we use information about HWE in the meta-analyses of genetic association studies? Int J Epidemiol 37: 136-146. 